\title{
MAGNETOHYDRODYNAMIC FLOW IN A TRUNCATED CONICAL ENCLOSURE
}

\author{
A. Bendjaghlouli ${ }^{1}$, B. Mahfoud ${ }^{2, *}$, D.E. Ameziani ${ }^{3}$
}

\begin{abstract}
The effect of an axial magnetic field on the flow produced by counter-rotation of the top and bottom disks in a truncated conical enclosure filled with a liquid metal is studied. The governing Navier-Stokes, and potential equations are solved by using the finite-volume method. It was observed that the Reynolds number is increased, the axisymmetric basic state loses stability and giving an asymmetric mode $\mathrm{m}=1$. It is also found that the primary thresholds $\mathrm{Re}_{\text {cr }}$ corresponding to the modes $\mathrm{m}=1$ increase with increasing of the Hartmann number (Ha). Finally, stability diagram (Re-Ha) has been established according to the numerical results of this investigation.
\end{abstract}

Keywords: Axisymmetric, Asymmetric, Counter-Rotation, Conical Enclosure, Magnetohydrodynamic

\section{INTRODUCTION}

The flows between two rotating disks were the subject of many studies. These flows, first studied by Batchelor [1] were given the name of Von Kármán swirling flows by Zandbergen and Dijkstra[2],and occurred frequently in geophysical and in industrial applications. The major characteristics of the flow are known to be determined by two dimensionless parameters: The aspect ratio $(\gamma=H / R)$ and the Reynolds number $\left(R e=\Omega R^{2} / v\right)$.

When a magnetic field is applied to a flow of an electrically conducting fluid, complex induction mechanisms occur and induced currents and magnetic field that are generated [3]. In the case of crystal growth, for example, magnetic fields are used to suppress the convective motion induced by the arising strong fluxes in order to control the flow in the melt, and consequently the crystal quality [4]. There are various studies of flow and heat transfer in a rotating system in the presence of a magnetic field [5-12]. We continue our previous study $[13,14,15]$ with attention has turned to the three-dimensional symmetry breaking of the basic state in a conical enclosure.

We have studied the three-dimensional forced convective magnetohydrodynamic flow in a truncated conical enclosure of radius $R$ (bottom) and $\mathrm{R}_{\mathrm{top}}=1 / 2 \mathrm{R}$ (top), having an aspect ratio $(\gamma=H / R=2)$, filled with a liquid metal characterized by a small Prandtl number $(P r=0.015)$ and submitted an axial magnetic field. The bottom disk is rotating with a constant angular velocity $\Omega$. Numerical results were obtained for various values of angular velocity ( $\Omega_{\text {top }}=1 / 4 \Omega, 1 / 2 \Omega, \Omega, 2 \Omega, 4 \Omega$ respectively) and for Hartmann numbers $H a=5,10,15$ and 20 . The objective is to determine critical Reynolds numbers for the transition from axisymmetric to nonaxisymmetric flow. The critical Reynolds number $R e_{\mathrm{cr}}$ are observed depending on the combination of the angular velocity of the top disk number and Hartmann numbers.

\section{MATHEMATICAL MODELLING Model}

The geometry under consideration is shown in Fig. 1. A liquid metal with a density $\rho$, a kinematic viscosity $v$ and an electrical conductivity $\sigma$, fills a truncated conical enclosure of radius $\mathrm{R}$ (bottom disk) and $\mathrm{R}_{\mathrm{top}}=1 / 2 \mathrm{R}$ (top disk) and height $\mathrm{H}(\gamma=\mathrm{H} / \mathrm{R}=2)$, submitted to an axial magnetic field $B$. The bottom disk is rotating with a constant angular velocity $\Omega$, while the top disk is in counter-rotating with various values of angular velocity $\left(\Omega_{\text {top }}=1 / 4 \Omega, 1 / 2 \Omega, \Omega, 2 \Omega, 4 \Omega\right.$ respectively). The walls of the truncated cone are electrically insulated. The induced magnetic field is negligible because the magnetic Reynolds number $R e_{\mathrm{m}}$ is much smaller than the unity. All the physical properties are taken as constant. 


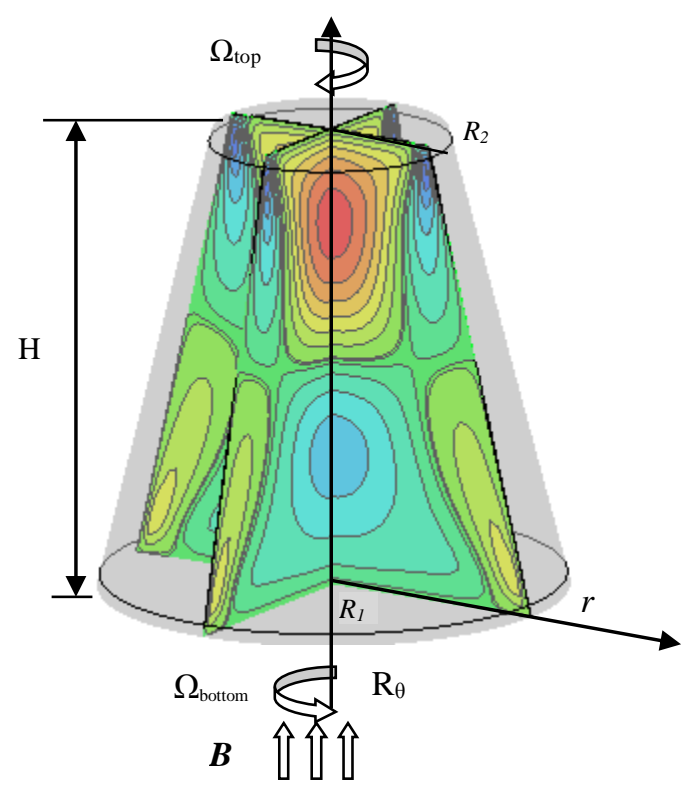

Figure. 1 Flow geometry and schematic of symmetries

\section{Governing Equations}

By neglecting the dissipation, and using $R, 1 / \Omega, \Omega R, \rho(\Omega R)^{2}$ and $\Omega R^{2} B$ as typical scales, respectively, for lengths, time, velocities, pressure and electric potential, the continuity, momentum and potential equations can be written, in a dimensionless form, as follows [13,14,15]:

$$
\begin{aligned}
& \nabla \mathrm{U}=0 \\
& \frac{\partial \mathbf{U}}{\partial \tau}+\mathbf{U} \cdot \nabla \mathbf{U}=-\nabla \mathrm{P}+\frac{1}{\operatorname{Re}} \nabla^{2} \mathbf{U}+\frac{\mathrm{Ha}^{2}}{\operatorname{Re}}\left(\mathbf{J} \mathbf{x} \mathbf{e}_{z}\right) \\
& \nabla^{2} \Phi=\nabla \cdot\left(\mathrm{U}_{x} \mathrm{e}_{z}\right) .
\end{aligned}
$$

$\mathrm{U}$ is the velocity vector $(u, v, w): u, v$ and $w$ for the radial, axial and azimuthal velocity components, respectively. $\mathbf{e}_{z}$ is the unit vector in the axial direction; $P$ is the dimensionless pressure.

The dimensionless physical parameters are:

The Reynolds number, $R e=\Omega R^{2} / v$ and the Hartmann number, $H a=B R \sqrt{\sigma / \rho v}$

The initial condition for each parametric case is that the fluid must be at rest. The bottom disk start their rotation with angular velocity $\Omega$ and the top disk is in counter-rotating with various values of angular velocity $\left(\Omega_{\text {top }}=1 / 4 \Omega, 1 / 2 \Omega, \Omega, 2 \Omega, 4 \Omega\right)$ respectively.

At $\tau=0$ :

$$
u=0, v=0, w=0, \Phi=0
$$

The boundary conditions, for $\tau>0$, are:

Sidewall electrically insulated:

$$
u=0, v=0, w=0, \frac{\partial \Phi}{\partial r}=0 \quad(0 \leq z \leq \gamma, z / 4-1 \leq r \leq 1-z / 4)
$$

At rotating bottom disk:

$$
u=0 \quad v=0, \quad w=r, \quad \frac{\partial \Phi}{\partial z}=0 \quad(z=0)
$$

At counter-rotating top disk: 


$$
u=0, v=0, w=-\alpha r, \frac{\partial \Phi}{\partial z}=0(z=\gamma)
$$

with $\alpha=1 / 4,1 / 2,1,2,4$ respectively, the periodicity condition:

$$
\begin{aligned}
& \mathrm{U}(r, \theta, z)=\mathrm{U}(r, \theta+2 \pi, z) \\
& P(r, \theta, z)=P(r, \theta+2 \pi, z)
\end{aligned}
$$

\section{NUMERICAL METHOD}

The governing equations (1)-(3), with the associated boundary conditions, are solved using the finitevolume method. The components of the velocity $(u, v$ and $w)$ are stored at the staggered locations, and the scalar quantities $(\mathrm{P}$ and $\Phi)$ are stored in the centre of these volumes. A fully implicit time marching scheme is used. The numerical procedure, which is SIMPLER [16], is used to handle the pressure-velocity coupling. For treatment of the diffusion terms in equations (2)-(3), central difference scheme is adopted. Convergence at a given time step is declared when the maximum relative change between two consecutive iteration levels fell below $10^{-5}$, for $u$, $v$, and $w$. At this stage, the steady state solution is obtained. At this stage, the steady state solution is obtained. The solution was obtained as follows:

- $\quad$ Equations (1) and (2) are first solved to get $\mathrm{P}, u, v$ and $w$.

- $\quad$ The potential $\Phi$ are subsequently computed from Eqs. (3) .

The different quantities associated with the set of equations, are discretised on non-uniform grids and solved on the $(r ; \theta ; z)$. For a properly resolved direct numerical simulation, especially in the case of MHD flow, the features of the specific flow, heat transfer and boundary layers must be considered. In particular, the increase of $\mathrm{Ha}$ results to thinner Hartmann layers at the walls normal to the magnetic field of thickness $\sim 1 / \mathrm{Ha}$. As a result, non-uniform grids were used for the simulation of the cases $H a=0$ to $H a=20$.

\section{RESULTS AND DISCUSSION Validation}

In the case without magnetic field, the present numerical results are compared with the previous numerical study of Escudier et al.[17], ,who carried numerical calculations for flow in a truncated cone generated by rotation of one endwall. In Fig.2, we have shown the reproduced streamlines for geometry with $\operatorname{Re}=1854$, for angle of inclination of container sidewall $(\alpha=-7)$.
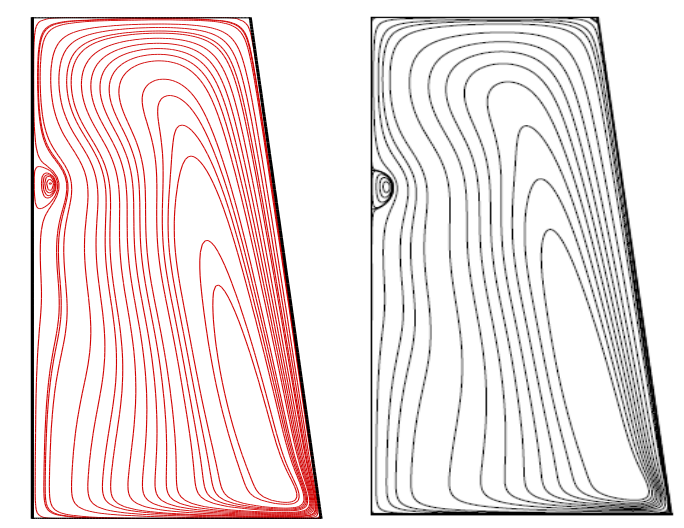

Figure 2. Comparison between present numerical results and numerical result of Escudier et al.[17]: Streamlines for convergent geometry with $\operatorname{Re}=1854$ and $\alpha=-7$.

\section{Basic State}

The basic flow consists of a dominant azimuthal shear layer produced by the counter-rotating end disks [1]. There are also second-order recirculation zones due to Ekman pumping in the area of each disk. The two zones of the meridional flow meet at the mid-plane and converge to form an inwardly directed radial jet. The configuration in basic state is invariant under rotations $\mathrm{R}_{\theta}$ about the $z$-axis. These symmetries are illustrated in Fig.1, which shows the 
plots of vertical velocity contours at $R e=300$. (basic state flow). We have remarked that $v$ is invariant under rotations $\mathrm{R}_{\theta}$ about the $z$-axis.

\section{Bifurcations without Magnetic Field $(\mathrm{Ha}=\mathbf{0})$}

Starting from the calculated $R e=500$ and increasing $\Omega_{\text {top }}$ gradually until the first asymmetric solution is observed up to $\Omega_{\text {top }}=2 \Omega$, the mode $m=1$ was shown in this case, and becomes stable at $\Omega_{\text {top }}=4 \Omega$ via a sequence of bifurcations. The vortices shown in Fig. 3 are confined near the sidewall and are manifested in the bulk as waviness in the shear layer. Fig. 3 shows that the maximum axial velocity is increased by increasing the angular velocity of the top disk. The bifurcation from the azimuthal mode $m=0$ (axisymmetric) shown in Fig. 3(a-c) to the steady state branch of mode $m=1$ (non-axisymmetric), occurs for $\Omega_{\text {top }}>1.8 \Omega$. The mode $m=1$ can be viewed as the result of the first instability of the equatorial shear layer produced by the counter-rotating disks.

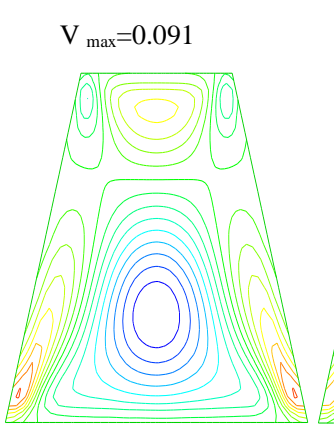

(a)

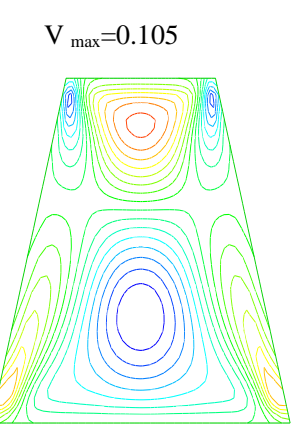

(b)

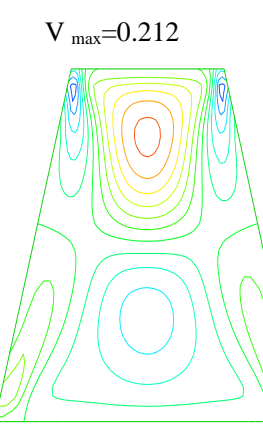

(c)

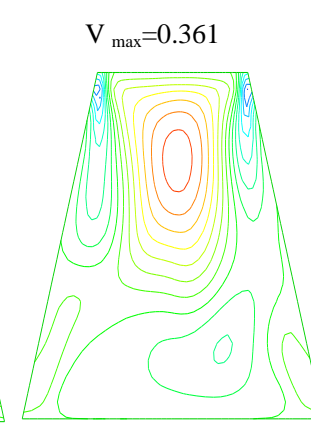

(d)

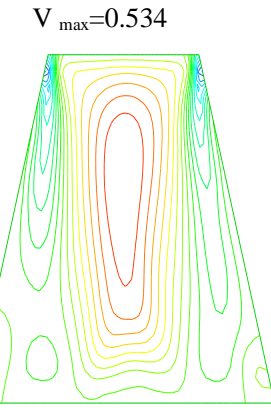

(e)

Figure 3. Iso-contours of axial velocity with $\mathrm{Re}=500$ and $\mathrm{Ha}=0$ (a) $\Omega_{\mathrm{top}}=1 / 4 \Omega$, (b) $\Omega_{\mathrm{top}}=1 / 2 \Omega$, (c) $\Omega_{\mathrm{top}}=\Omega$, (d) $\Omega_{\mathrm{top}}=2 \Omega$, (e) $\Omega_{\mathrm{top}}=4 \Omega$.

\section{Bifurcations with Magnetic Field $(\mathrm{Ha} \neq 0)$}

When an axial magnetic field is imposed, some interesting changes are observed in the motion of the fluid. In the case of turbulent flow, the presence of the magnetic field results in the stabilization of the flow turning it to laminar and axisymmetric.

As it is already mentioned, for the case $R e=500$ and $\Omega_{t o p}=2 \Omega$, the flow is fully asymmetric. For High values of Ha number, the asymmetric flow becomes axisymmetric. Fig. 4 shows the axial velocity at meridional plane $r=0$, for $R e=500$, and for different values of angular velocity $\left(\Omega_{\mathrm{top}}=1 / 4 \Omega, 1 / 2 \Omega, \Omega, 2 \Omega, 4 \Omega\right.$ respectively). We can see It is easily observed at this value of Hartmann number that the flow remains axisymmetric whatever the rotation rate. As it concerns the flow structure the magnetic field suppresses the fluid motion and the axial velocity decreases with increasing Hartmann number (see, Fig.5). We can see the decrease of the axial velocity when the Hartmann number increases. The regions located just under the rotating disk and just above the bottom counter-rotating disk correspond to a concentration of the electric current lines perpendicular to the magnetic field B. As a result, in these locations the Lorentz force is large. When the Hartmann number increases, the Ekman layer characterised by the equilibrium between the centrifugal and viscous forces is progressively replaced by the Hartmann layer characterised by equilibrium between electromagnetic and viscous forces [8]. This is shown in Fig. 5 where iso-contours of axial velocity at $z=0.5$ (bottom), $z=1$ (middle), and $z=1.5$ (top), for the cases $\Omega_{\text {top }}=\Omega$ and various values Hartmann number $H a=5,10,15$ and 20 are compared.

\section{Stability Diagram}

For the counter-rotating end disks case with $\Omega_{\mathrm{top}}=\Omega$, the stability diagram presented in Fig. 6 is constructed on $\left(R e_{\mathrm{cr}}-H a\right)$ plane. It is observed in this figure the evolution of the primary thresholds, $R e_{\mathrm{cr}}$ corresponding to the mode $m=1$ as a function of $H a$. We have shown that the order of appearance of these mode changes when $R e$ is increased (the contour plots of the vertical velocity $v$ in the horizontal plane, for the corresponding mode, are given with respect to this order). The only effect of increasing $H a$ is a continuous increase in $R e_{\mathrm{cr}}$. In this diagram, we also give the iso-contours of the vertical velocity in the $z=1$ plane for the different branches of solutions (above the curve) at $(R e=450, H a=5) ;(R e=700, H a=10)$; $(R e=900, H a=15)$. For $H a=5$ the mode $m=1$ appears for at $R e=$ 405. For $H a=10$ the mode $m=1$ appears at $R e=560$ and for $H a=15$ the $m=1$ appears at $R e=810$. 


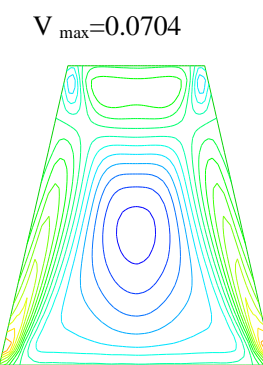

(a)

F

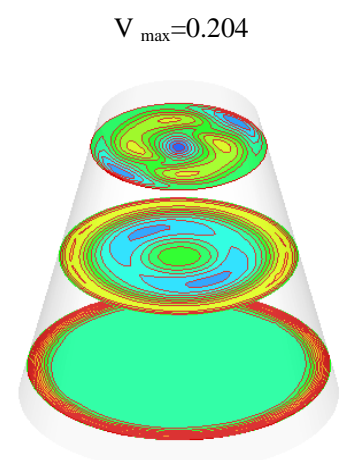

$\mathrm{Ha}=5$
$\mathrm{V}_{\text {max }}=0.07081$

$\mathrm{V}_{\max }=0.1838$

(c)

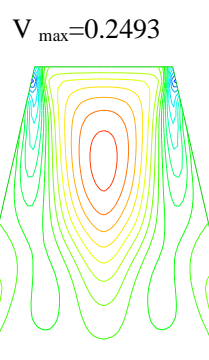

(d)
$\mathrm{V}_{\text {max }}=0.4023$

(e)

(d) $\Omega_{\text {top }}=2 \Omega$, (e) $\Omega_{\text {top }}=4 \Omega$.
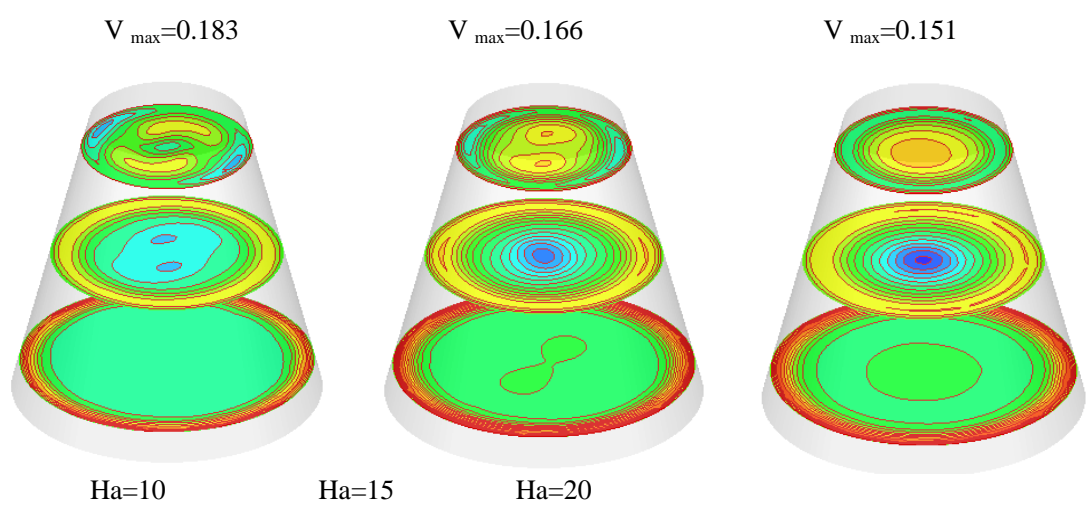

Figure 5. Iso-contours of axial velocity at $z=0.5$ (bottom), $z=1$ (middle), and $z=1.5$ (top), for the case of $\operatorname{Re}$ $=1000\left(\Omega_{\mathrm{top}}=\Omega\right)$. Asymmetric flow for three cases: $\mathrm{Ha}=5, \mathrm{Ha}=10, \mathrm{Ha}=15$; axisymmetric basic flow for the case of $\mathrm{Ha}=20$.

For a moderate Hartmann number $(H a=20)$, the flow sets in with the mode $m=1$ for $R e=1050$. Under the curve, we present the structure of axisymmetric contours of vertical velocity at meridional plane $r=0$, which correspond to an axisymmetric solution $(m=0)$ in case of $R e=300$ with $H a=10$ and 20. Finally, all the primary thresholds increase with the Hartmann number, confirming the phenomenon of flow stabilization by the application of the magnetic field.

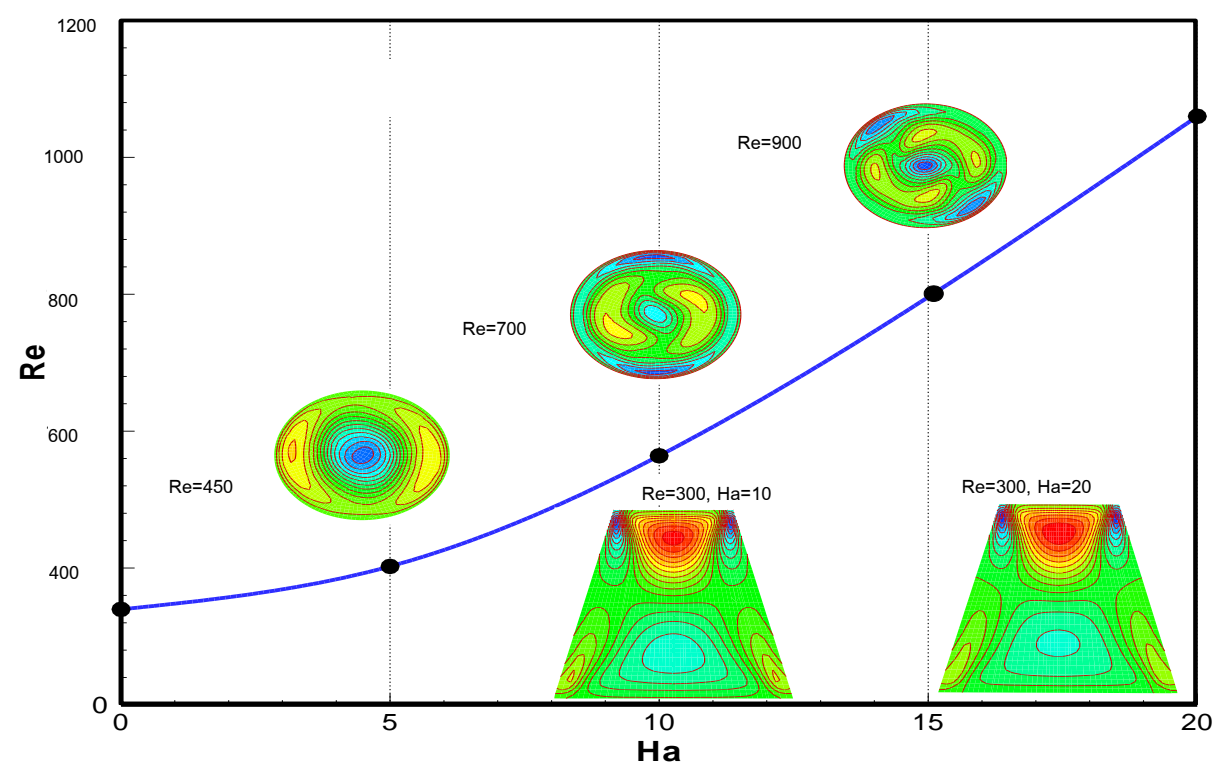

Figure 6. Stability diagram in the $\left(R e_{\mathrm{cr}}-H a\right)$ plane for $\left(\Omega_{\mathrm{top}}=\Omega\right)$. Evolution of the primary thresholds $R e_{\mathrm{cr}}$ corresponding to the modes $m=1$, as a function of $H a$. 


\section{CONCLUSION}

Flow produced by counter-rotation of the top and bottom disks in a conical enclosure having an aspect ratio equals to 2 , filled with a liquid metal has been numerically studied. The bottom disk is rotating with a constant angular velocity $\Omega$, while the top disk is in counter-rotating with various values of angular velocity. The study is divided into two parts, with and without magnetic field. The finite volume method has been used to solve numerically the transport equations. The effects of both the magnetic field and angular velocity have been studied. The main results obtained in this study are as follows:

- The developed code in this study was validated with the results found in the literature, and a good agreement has been obtained.

- It was observed that when the Reynolds number is increased, the axisymmetric basic state loses stability and different complex flows appear successively and the structure of the flows is dominated by the mode $m=1$.

- The present results show that the increase of the Hartmann number causes the increase of the critical Reynolds number. Therefore, the increase in the values of $\mathrm{Ha}$, suppresses the fluid motion and reduce the axial velocity.

- It is shown when the magnitude of the magnetic field (Ha) exceeds a certain value the steady three-dimensional flow obtained for large Re, due to the counter-rotation mechanism tends to be axisymmetric flow and confirming the phenomenon of flow stabilization by the application of the magnetic field.

\section{NOMENCLATURE}

B magnitude of the external magnetic field

$H$ height of the cylinder

$P$ dimensionless pressure

$R$ radius of the cylinder

$r, \theta, z$ dimensionless spatial coordinates

$\mathrm{U}$ dimensionless velocity vector

Greek symbols

$\alpha$ thermal diffusivity of the fluid

$\beta$ thermal expansion coefficient

$\gamma$ aspect ratio

$\Theta$ dimensionless temperature

$\rho$ density of the fluid

$\sigma$ electric conductivity

$\Phi$ dimensionless electric potential

$\Omega$ angular velocity

$\tau$ dimensionless time

Subscripts

cr critical value.
(Tesla)

(m)

( - )

(m)

(m)

$(-)$

$\left(\mathrm{m}^{2} / \mathrm{s}\right)$

$(1 / \mathrm{K})$

$(-)$

$(-)$

$\left(\mathrm{kg} / \mathrm{m}^{3}\right)$

$(\Omega / \mathrm{m})$

$(-)$

$(\mathrm{rad} / \mathrm{s})$

$(-)$

\section{REFERENCES}

[1] Batchelor, G.K. (1951). Note on a class of solutions of the Navier-Stokes equations representing steady rotationally-symmetric flow. Quart. J. Mech. Appl. Math., 4(1), 29-41.

[2] Zandbergen, P.J., Dijkstra D. (2004). Von Kármán swirling flows. Annu. Rev. Fluid Mech., (19), 465-491.

[3] Bourgoin, M., Odier, P., Pinton, J.F., Ricard, Y. (2004). An iterative study of time independent induction effects in magnetohydrodynamics. Phys. Fluids, 16(7), 2529-2547.

[4] Touihri, R., Ben Hadid, H., Henry, D. (1999). On the onset of convective instabilities in cylindrical cavities heated from below. II. Effect of a magnetic field. Phys. Fluids, 11(8), 2089-2100.

[5] Sheikholeslami, M., Hatami, M., Ganji, DD. (2014). Nanofluid flow and heat transfer in a rotating system in the presence of a magnetic field. Journal of Molecular liquids, 190, 112-120.

[6] Sheikholeslami, M., Hatami, M., Domairry, G. (2015). Numerical simulation of two phase unsteady nanofluid flow and heat transfer between parallel plates in presence of time dependent magnetic field. Journal of the Taiwan Institute of Chemical Engineers, 46,43-50.

[7] Esfahani, J.A., Kianifar, A., Rashidi, S., Bovand, M., Shirvan, K. M. (2015). Control of Wake and Vortex Shedding Behind Solid Circular Obstacle by Magnetohydrodynamics". Journal of Thermal Engineering, 7(1), 593-597. 
[8] Hussein, A. K., Hussain, S. H. (2015).Characteristics of Magnetohydrodynamic Mixed Convection in a Parallel Motion Two-Sided Lid-Driven Differentially Heated Parallelogrammic Cavity with Various Skew Angles. Journal of Thermal Engineering, 3(1),221-235.

[9] Esfahani, J.A., Kianifar, A., Rashidi, S., Bovand, M., Shirvan, K. M. (2015). Control of Wake and Vortex Shedding Behind Solid Circular Obstacle by Magnetohydrodynamics. Journal of Thermal Engineering, 7(1),593-597.

[10] Hussein, A. K., Hussain, S. H. (2015) .Characteristics of Magnetohydrodynamic Mixed Convection in a Parallel Motion Two-Sided Lid-Driven Differentially Heated Parallelogrammic Cavity with Various Skew Angles. Journal of Thermal Volume, 3(1), 221-235.

[11] Dash, S., Singh, N. (2017).Study of Axisymmetric Nature in 3-D Swirling Flow in a Cylindrical Annulus with a Top Rotating Lid under the Influence of Axial Temperature Gradient or Axial Magnetic Field. Journal of Thermal Engineering, 6(3),1588-1606.

[12] Geridönmez, B. P. (2018). Numerical Simulation of Natural Convection in a Porous Cavity Filled With Ferrofluid in Presence of Magnetic Source. Journal of Thermal Engineering, 2(4), 1756-1769

[13]Mahfoud, B., Bendjagloli, A., Bessaïh R. (2016).Magneto-hydrodynamic co-rotating flow in a vertical cylindrical container. Numerical Heat Transfer, Part A, 69,1051-106.

[14]Mahfoud, B., Bessaïh, R. (2016).Magnetohydrodynamic counter-rotating flow in a cylindrical cavity. International Journal of Heat and Mass Transfer, 93,175-185.

[15] Mahfoud, B. Bendjaghloli, A. (2018). Natural convection of a nanofluid in a conical container" Journal of Thermal Engineering, 4,1713-1723.

[16] Patankar, S. (1980). Numerical heat transfer and fluid flow. CRC press.

[17] Escudier, M.P., O’Leary J. , Poole, R.J. (2007). Flow produced in a conical container by a rotating endwall," International Journal of Heat and Fluid Flow, 28,1418-1428. 